\title{
Visualization of skin morphology and microcirculation with high frequency ultrasound and dual-wavelength photoacoustic microscope
}

Yoshifumi Saijo, Taiichiro Ida, Hideaki Iwazaki, Jun Miyajima, Huabin Tang, et al.

Yoshifumi Saijo, Taiichiro Ida, Hideaki Iwazaki, Jun Miyajima, Huabin Tang, Ryo Shintate, Kanta Sato, Tatsuki Hiratsuka, Shin Yoshizawa, Shinlchiro Umemura, "Visualization of skin morphology and microcirculation with high frequency ultrasound and dual-wavelength photoacoustic microscope," Proc. SPIE 10878, Photons Plus Ultrasound: Imaging and Sensing 2019, 108783E (27 February 2019); doi: 10.1117/12.2508599

SPIE. Event: SPIE BiOS, 2019, San Francisco, California, United States 


\title{
Visualization of skin morphology and microcirculation with high frequency ultrasound and dual-wavelength photoacoustic microscope
}

\author{
Yoshifumi Saijo $^{\mathrm{a}^{*}}$, Taiichiro Ida ${ }^{\mathrm{b}}$, Hideaki Iwazaki ${ }^{\mathrm{b}}$, Jun Miyajima ${ }^{\mathrm{b}}$, Huabin Tang ${ }^{\mathrm{b}}$, Ryo Shintate ${ }^{\mathrm{a}}$, \\ Kanta Sato $^{a}$, Tatsuki Hiratsuka ${ }^{a}$, Shin Yoshizawa ${ }^{a}$, ShinIchiro Umemurai ${ }^{a}$ \\ ${ }^{a}$ Tohoku University, 6-6-05 Aramaki Aza Aoba, Aoba-ku, Sendai, JAPAN 980-8579; \\ ${ }^{\mathrm{b}}$ Advantest Corporation, 1-5, Shin-tone, Kazo, JAPAN 349-1158
}

\begin{abstract}
Skin aging is characterized by color and wrinkle caused by degeneration of collagen and elastin in the dermis. Recently, the volume, diameter and branching of the micro vessels in the skin are proved to affect these biomechanical changes. Thus, high resolution imaging for both micro structure and micro vessels of the skin is desired. In the present study, dual-wavelength photoacoustic microscope (PAM) combined with high frequency ultrasound (HFUS) is developed to visualize both the morphology and microcirculation of the skin.

Two Nd:YAG laser light sources with the wavelength of 532/556 nm, pulse width of 1.2/3.6 ns, pulse energy of 16 $\mu \mathrm{J} /$ pulse and repetition rate of $1 \mathrm{kHz}$ were equipped in the HFUS-PAM system. The optical fiber for laser delivery was inserted through the center hole of the concave ultrasound transducer with the central frequency of $75 \mathrm{MHz}$. Both HFUS and PA signals were acquired at the sampling rate of $500 \mathrm{MHz}$ and the resolution of 12 bits. The transducer was scanned by voice coil actuators to obtain 3D dataset of HFUS and PA signals. Oxygen saturation of the micro circulation was calculated by the PA signals alternately obtained at $532 \mathrm{~nm}$ and $556 \mathrm{~nm}$. 3D image of the layered structure and the micro vessels representing oxygen saturation in the $6 \mathrm{~mm} \times 6 \mathrm{~mm} \times 3 \mathrm{~mm}$ volume of the skin was successfully obtained with the system. HFUS-PAM will provide important information of skin morphology and microcirculation for assessment of skin aging.
\end{abstract}

Keywords: Photoacoustic microscopy, high frequency ultrasound, skin, morphology, microcirculation

\section{INTRODUCTION}

In the era of aging society, much attention is paid to skin aging and precise evaluation method of skin becomes more important. Not only surface color or wrinkle, biomechanics of dermis consisting of collagen, elastin, sebaceous glands and micro vessels, contributes to skin aging. For imaging of dermis, optical imaging modalities such as scanning laser microscopy or optical coherence tomography don't have enough penetration depth to observe whole layer of dermis. High frequency ultrasound (HFUS) has realized high resolution skin imaging to observe whole layer of the dermis and to measure the viscoelasticity of dermis and the volume of sebaceous glands. HFUS also provides semi-quantitative information of collagen quantity and quality by intensity of reflected ultrasound. Biomechanics of the dermis is assessed by elastography technique with HFUS.

Recently, the volume, diameter and branching of the micro vessels in the dermis are proved to affect the skin lesions. Precise 3D visualization of blood vessels by optical clearing technique that makes skin tissue transparent, shows increase in number and density of blood vessels in pigmented skin [1]. Thus, in vivo visualization of blood vessels in the skin is important to assess skin aging. Photoacoustic (PA) imaging is rapidly developing imaging modality in these ten years. PA imaging is based on PA phenomenon and it typically enables high resolution imaging of blood vessels with deeper penetration depth than conventional optical imaging. Distribution of small blood vessels are closely associated with skin structure such as hair follicles or sebaceous glands. Thus, simultaneous imaging of skin morphology and microcirculation is important. However, most high resolution PA imaging systems capable of small vessel imaging, don't visualize structure of the tissue. In the present study, PA and HFUS combined microscope is proposed for visualization of three-dimensional (3D) structure of micro vessels and the association with tissue structures in dermis.

*saijo@tohoku.ac.jp; phone 8122 795-7148; fax 8122 795-7149

Photons Plus Ultrasound: Imaging and Sensing 2019, edited by Alexander A. Oraevsky, Lihong V. Wang, Proc. of SPIE Vol. 10878, 108783E - (C) 2019 SPIE · CCC code: 1605-7422/19/\$18 · doi: 10.1117/12.2508599 


\section{METHODS}

\subsection{System configuration}

Figure 1 shows the block diagram of the HFUS-PAM system. Two Nd:YAG laser light sources were equipped. One was with the wavelength of $532 \mathrm{~nm}$ and the pulse width of $1.2 \mathrm{~ns}$. The other was with the wavelength of $556 \mathrm{~nm}$ and the pulse width of $3.6 \mathrm{~ns}$. In both light sources, the pulse energy was $16 \mu \mathrm{J} / \mathrm{pulse}$ and the repetition was $2 \mathrm{kHz}$. The optical fiber for laser delivery was inserted through the center hole of the concave ultrasound transducer with the central frequency of $50 \mathrm{MHz}$. The design was based on our previous studies [2,3]. The diameter of the optical fiber was $105 \mu \mathrm{m}$ and the diameter of the transducer was $3.6 \mathrm{~mm}$. Both HFUS and PA signals were acquired at the sampling rate of 500 $\mathrm{MHz}$ and the resolution of 12 bits. The transducer was scanned by voice coil actuators to obtain 3D dataset of HFUS and PA signals. The interval of the scan step was either 15 or $30 \mu \mathrm{m}$ resulting the scan area of $6 \times 3 \mathrm{~mm}$ or $6 \times 6 \mathrm{~mm}$.

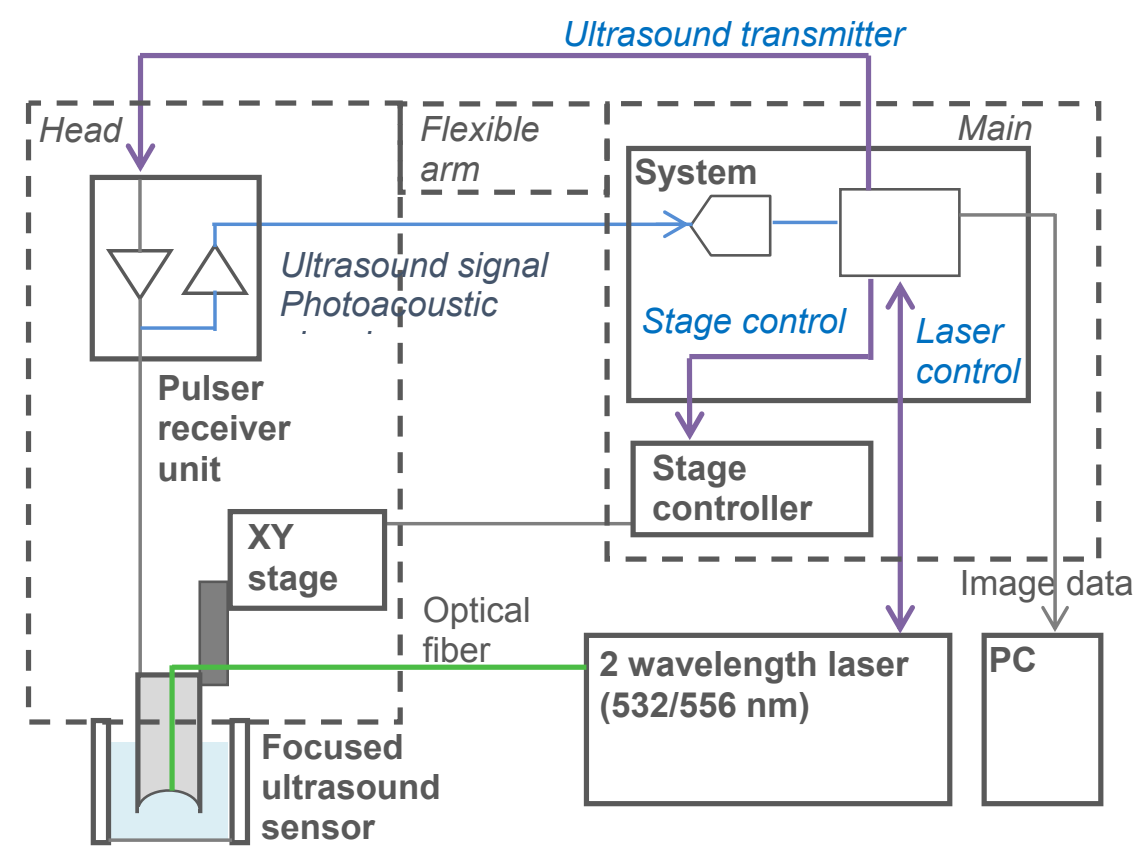

Figure 1. Block diagram of the HFUS-PAM system with dual-wavelength light sources

\subsection{Oxygen saturation}

Oxygen saturation of the micro circulation was generally calculated by following equation.

$$
\mathrm{SaO}_{2}=\frac{\mathrm{C}_{\mathrm{HbO} 2}}{\mathrm{C}_{\mathrm{HbO} 2}+\boldsymbol{C}_{\mathrm{Hb}}} \times 100
$$

( $\mathrm{SaO}_{2}$ : oxygen saturation, $\boldsymbol{C}_{\boldsymbol{H b o} 2}$ : concentration of the oxy-hemoglobin $\left(\mathrm{HbO}_{2}\right), \boldsymbol{C}_{\boldsymbol{H b}}$ : concentration of the deoxyhemoglobin $(\mathrm{Hb}))$

In the present study, local oxygen saturation was calculated by the PA signals alternately obtained at $532 \mathrm{~nm}$ and $556 \mathrm{~nm}$ by following equation [4]. 


$$
\boldsymbol{S a O}_{2}=\frac{\frac{\boldsymbol{p}_{532}}{\boldsymbol{p}_{556}} \cdot \frac{\Phi_{556}}{\boldsymbol{\Phi}_{532}} \cdot \mu_{556}-\mu_{532}}{\frac{\boldsymbol{p}_{532}}{\boldsymbol{p}_{556}} \cdot \frac{\Phi_{556}}{\Phi_{532}} \cdot \delta \mu_{556}-\delta \mu_{532}}
$$

( $\boldsymbol{\mu}_{532}$ : optical absorption coefficient of $\mathrm{Hb}$ at the wavelength of $532 \mathrm{~nm}, \boldsymbol{\mu}_{556}$ : optical absorption coefficient of $\mathrm{Hb}$ at the wavelength of $556 \mathrm{~nm}, \boldsymbol{\Phi}_{532}$ : optical fluence at the wavelength of $532 \mathrm{~nm}, \boldsymbol{\Phi}_{556}$ : optical fluence at the wavelength of 556 $\mathrm{nm}, \boldsymbol{\delta} \boldsymbol{\mu}_{532}$ : difference between the optical absorption coefficients of $\mathrm{Hb}$ and $\mathrm{HbO}_{2}$ at the wavelength of $532 \mathrm{~nm}, \boldsymbol{\delta} \boldsymbol{\mu}_{556}$ : difference between the optical absorption coefficients of $\mathrm{Hb}$ and $\mathrm{HbO}_{2}$ at the wavelength of $556 \mathrm{~nm}$ )

Although wavelength dependent scattering could cause errors in calculating the $\mathbf{S a O}_{2}$ values, wavelengths used in this system were very close. Therefore, the difference in path length was negligible in this system.

\subsection{Phantom study}

The resolution of the HFUS-PAM system was evaluated by visualization of a carbon wire with the diameter of $7 \mu \mathrm{m}$. Calculation of oxygen saturation was evaluated by a tube phantom filled with horse blood and a tube phantom filled with horse blood after oxygen reduction.

\subsection{Measurement of human subjects}

Total number of 40 female subjects were involved in the measurement with HFUS-PAM. The skin vasculature and morphology in forehead, cheek, upper arm, finger and sole were visualized by the system. The study protocol was approved by the Ethics Committee on Research involving Human Subjects in Graduate School of Engineering, Tohoku University. Informed consent was obtained from all the subjects.

\section{RESULTS}

\subsection{Resolution of the HFUS-PAM system}

Figure 2 shows the received PA signal in (a) time domain and (b) frequency domain. The signal was sharp and the effective frequency range covered $10-84 \mathrm{MHz}(-6 \mathrm{~dB})$.
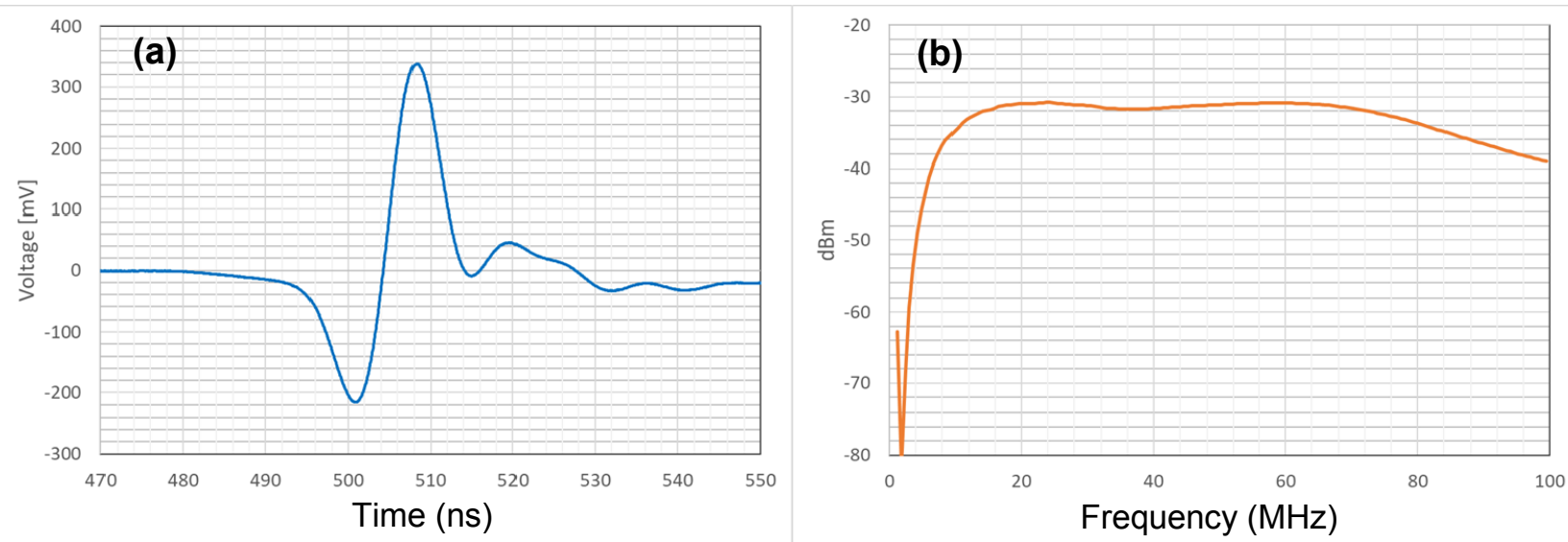

Figure 2. PA signal in (a) time domain and (b) frequency domain 
Figure 3 shows the graphs showing (a) horizontal resolution and (b) axial resolution. From these graphs, the horizontal resolution was found to be $24 \mu \mathrm{m}$ and the axial resolution was found to be $16 \mu \mathrm{m}$.
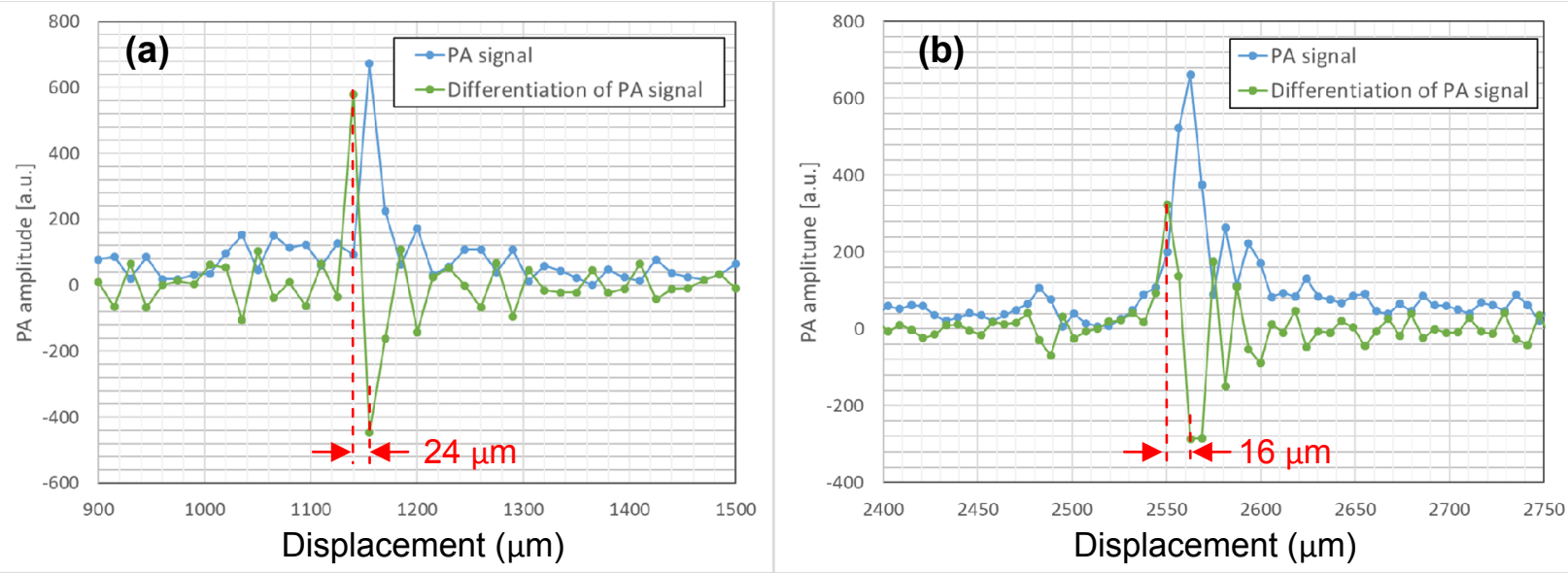

Figure 3. Graphs showing (a) horizontal resolution and (b) axial resolution

\subsection{Oxygen saturation measured with tube phantom}

Figure 4 shows the appearance of the tube phantom (a) with intact horse blood, (b) with reduced horse blood, calculated oxygen saturation of the tube phantom (c) with intact horse blood, (d) with reduced horse blood measured with HFUSPAM. The accuracy of the oxygen saturation with the system was validated by the results.

(a)

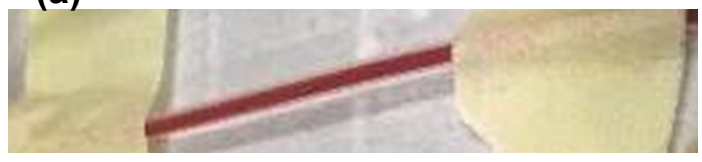

(b)

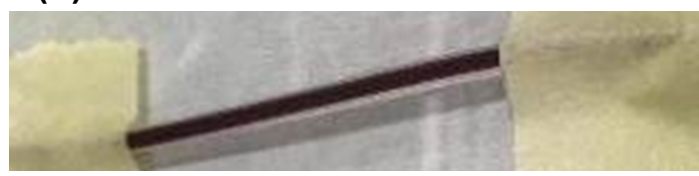

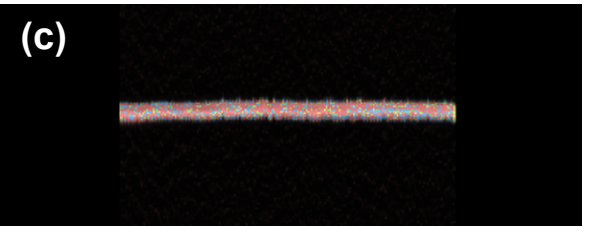

(d)

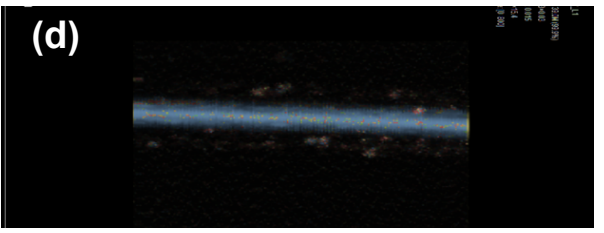

Figure 4. Appearance of the tube phantom (a) with intact horse blood, (b) with reduced horse blood, calculated oxygen saturation of the tube phantom (c) with intact horse blood, (d) with reduced horse blood measured with HFUS-PAM 


\subsection{Images of human subjects}

Figure 5 shows the examples of HFUS-PAM images taken in the human subjects. Figure 5 (a) is the bird's eye image and Figure 5 (b) is the tomographic image of finger. Small vessels underlying finger print are clearly observed. Figure 5 (c) is the bird's eye image and Figure 5 (d) is the tomographic image of cheek. Small vessels surrounding the hair follicles are clearly observed.
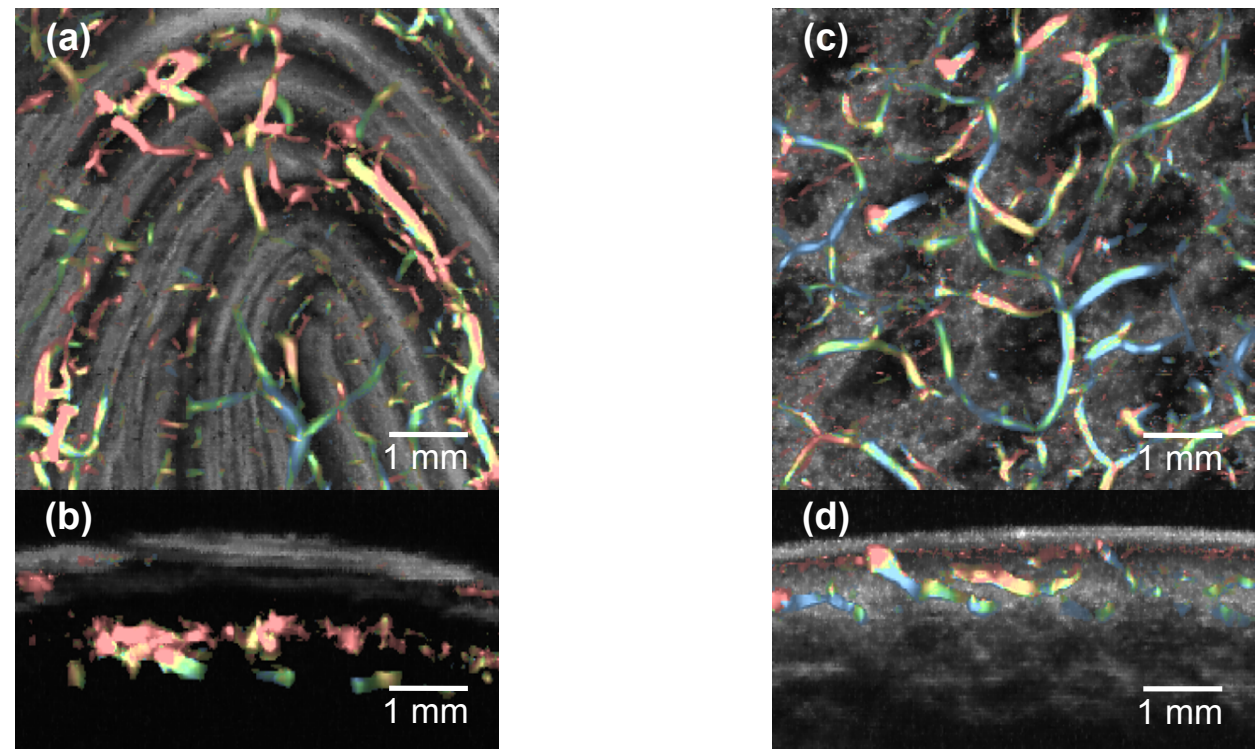

Figure 5. Examples of HFUS-PAM images. (a) bird's eye image, (b) tomographic image of finger, (c) bird's eye image and (d) tomographic image of cheek

\section{DISCUSSION}

\subsection{Performance of the HFUS-PAM system}

The HFUS-PAM system achieved horizontal resolution of $24 \mu \mathrm{m}$ and the axial resolution of $16 \mu \mathrm{m}$. Prior to the development of this system, the resolution was aimed to $30 \mu \mathrm{m}$ because most vascular network was structured with vessels thicker than $30 \mu \mathrm{m}$ in transparent skin tissue. Thus, the resolution was considered to satisfy in vivo visualization of vascular network of skin. The penetration depth of PA signal was approximately $2 \mathrm{~mm}$ in measurement of human subjects. PA signal deeper than $2 \mathrm{~mm}$ was too low to produce image. As the thickness of the dermis is less than $2 \mathrm{~mm}$, the penetration depth is enough to observe the whole layer of dermis.

In contrast to that most PA systems obtain only PA image of vessels, this HFUS-PAM system can obtain both HFUS and PA images. The advantage of the system was to obtain vessel image by PAM superimposed on morphological image by HFUS. Especially, vessels surrounding hair follicle was clearly observed in HFUS-PAM image of cheek.

Dual-wavelength $(532 / 556 \mathrm{~nm})$ light source was equipped in the HFUS-PAM system. Oxygen saturation is calculated on assumption that ratio of optical absorption is proportional to PA signal intensity at each wavelength. The accuracy of the calculation was validated by tube phantom filled with horse blood with and without oxygen reduction. However, due to the different optical absorption at each wavelength in skin tissue, error should be considered in vivo measurement.

\subsection{Future implications}

Skin pigmentation is one of the features of skin aging and is known to associated with increased vessel by conventional dermoscopy findings [5]. Recently, imaging of vasculature using tissue clearing method $[1,6]$ or optical coherent tomography [7] are reported. The HFUS-PAM system directly observes the skin vasculature in non-invasive manner and the system may contribute serial measurement of skin vasculature after treatment or cosmetics. 


\section{CONCLUSIONS}

In the present study, dual-wavelength photoacoustic microscope (PAM) combined with high frequency ultrasound (HFUS) was developed to visualize both the three-dimensional (3D) morphology and microcirculation of the skin. 3D image of the layered structure and the micro vessels representing oxygen saturation in the $6 \mathrm{~mm} \times 6 \mathrm{~mm} \times 3 \mathrm{~mm}$ volume of the skin was successfully obtained with the system. HFUS-PAM will provide important information of skin morphology and microcirculation for assessment of skin aging.

\section{REFERENCES}

[1] Shibata, T., Kajiya, K., Sato, K., Yoon, J., Kang, H. Y., "3D microvascular analysis reveals irregularly branching blood vessels in the hyperpigmented skin of solar lentigo," Pigment Cell. Melanoma Res. 31(6):725727 (2018).

[2] Izumi. T., Sato, M., Yabe, Y., Hagiwara, Y., Saijo, Y., "Ultrasonic and photoacoustic imaging of knee joints in normal and osteoarthritis rats," Conf. Proc. 35th IEEE Eng. Med. Biol. Soc. 1116-9 (2013).

[3] Yamazaki, R., Ogasawara, K., Fujiwara, M., Kobayashi, K., Saijo, Y., "Macrophage with gold nanorod visualized by optical-resolution and acoustic-resolution photoacoustic microscopes," Conf. Proc. 37th IEEE Eng. Med. Biol. Soc. 2387-90 (2015).

[4] Perekatova, V., Subochev, P., Kleshnin, M., Turchin, I., "Optimal wavelengths for optoacoustic measurements of blood oxygen saturation in biological tissues," Biomed. Opt. Express. 7(10): 3979-3995 (2016).

[5] Togawa, Y., "Review of vasculature visualized on dermoscopy," J. Dermatol. 44(5):525-532 (2017).

[6] Hasegawa, K., Fujiwara, R., Sato, K., Park, J. Y., Kim, S. J., Kim, M., Kang, H.Y., "Increased blood flow and vasculature in solar lentigo," J. Dermatol. 43(10):1209-1213 (2016).

[7] Hara, Y., Yamashita, T., Kikuchi, K., Kubo, Y., Katagiri, C., Kajiya, K., Saeki, S., "Visualization of age-related vascular alterations in facial skin using optical coherence tomography-based angiography," J. Dermatol. Sci. 90(1):96-98 (2018). 